\title{
Laser-induced acoustic generation for buried object detection
}

\author{
S. W. McKnight ${ }^{a}$, C. A. DiMarzio ${ }^{a}$, W. Li ${ }^{a}$, R.A. Roy ${ }^{b}$ \\ ${ }^{a}$ Center for Electromagnetics Research, Northeastern University, Boston, MA 02115 \\ ${ }^{b}$ Department of Aerospace and Mechanical Engineering, Boston University, Boston, MA 02215
}

\begin{abstract}
Mechanisms for the production of acoustic energy in soil by pulsed $\mathrm{CO}_{2}$ laser excitation of the surface are reported. When the laser pulse in unfocused with a spot size about $1 \mathrm{~cm}$ in diameter, a single narrow acoustic pulse is observed with a spectral content near the detector limit of $100 \mathrm{kHz}$ and a velocity of $255 \mathrm{~m} / \mathrm{s}$, close to the speed of sound in air. When the laser is focused to a spot size on the order of $1 \mathrm{~mm}$ diameter, the audible acoustic intensity in greatly increased and we observe a second broad acoustic feature. This feature has a much lower frequency (near $3 \mathrm{kHz}$ ) and velocity $(75 \mathrm{~m} / \mathrm{s})$. We have tentative identified the fast mode as a normal compressive mode and the slow mode as a Biot slow-wave. A study of visible light emission when the focused $\mathrm{CO}_{2}$ laser beam strikes the sand surface indicates ionized nitrogen, oxygen, and silicon are present. This implies that the mechanism for sound production with the focused beam involves ionization by the optical electric field, expansion, and subsequence collapse of the air. The mechanism for sound production by the unfocused beam, which produces better imaging of underground objects, appears to be quite different.
\end{abstract}

Keywords: laser-induced, acoustic, landmine detection, Biot waves, porous media

\section{INTRODUCTION}

The use of high-frequency $(\sim 30 \mathrm{kHz})$ acoustic waves produced by a pulsed $\mathrm{CO}_{2}$ incident on the surface of dry sand for imaging of shallow buried objects such as anti-personnel landmines has been demonstrated in the laboratory ${ }^{1,2}$. The mechanisms for the conversion of the optical pulses into sound, however, is poorly understood. Since the optimization of the process of optical to acoustic conversion in a porous media depends on understanding and modeling the physical processes, we have initiated an experimental investigation of the behavior of the acoustic modes created in soil under different laser pulse focusing conditions. For a broad, unfocused laser spot, modeling the soil as a uniform effective medium as in Figure la may be appropriate. If the laser is focused to a small spot comparable to the size of the sand grains as in Figure 1b, a more complex calculation may be necessary which takes into account the random position and orientation of the sand grains and the intervening air spaces. In addition, under focused beam conditions the optical field intensity can become very large, leading to different physical effects. We have observed

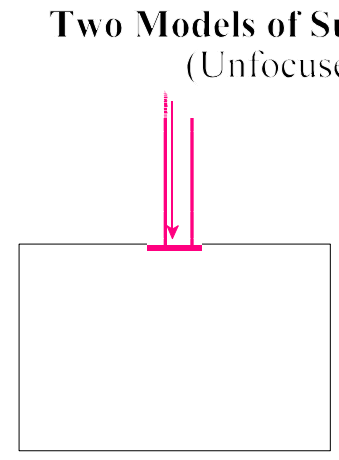

Effective Medium

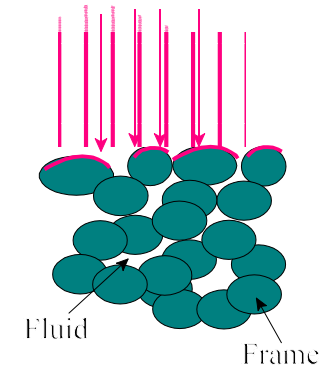

Random Porous Medium

Figure 1 Conceptual models of optical-to-acoustic conversion in soil. a) An effective medium approximation may be adequate when the laser spot is much larger than the structure of the soil, b) The details of the interaction of the light with the discrete particles of the poro-elastic medium may be critical when the beam size is comparable to the particle size.

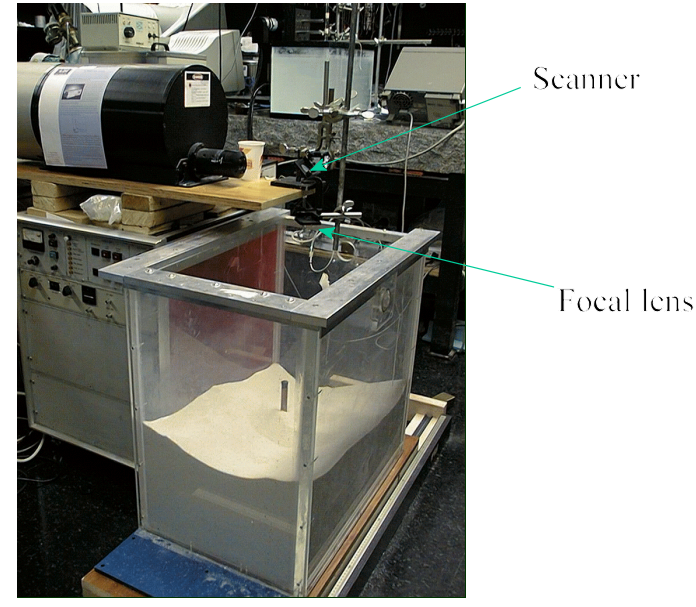

Figure 2 Photograph of experimental setup. The pulsed $\mathrm{CO}_{2}$ is at top left and the experimental sandbox is below. Objects to be detected or hydrophones to study the laser-to-acoustic coupling are buried in the sand. 
that focusing the laser beam to a spot size on the order of $1 \mathrm{~mm}$ in diameter greatly increases the audible acoustic sound associated with the laser pulse and, in addition, can cause the production of a flash of visible light at the point where the infrared $\mathrm{CO}_{2}$ pulse impinges on the sand. We have studied the time-dependence of the acoustic pulse at various depths and angles in the soil for both focused and unfocused laser pulses. For unfocused pulses we find a single acoustic mode with a velocity slightly less than the speed of sound in air with a directionality perpendicular to the soil surface. With a focused beam we detect two distinct acoustic features in the time trace: a sharp pulse with a velocity and spectral content similar to that achieved with the unfocused beam, and, in addition, a second broad pulse with a much lower frequency content which propagates at a slower velocity. We suggest that these two modes may be related to the two Biot modes allowed in poro-elastic media ${ }^{3}$.

\section{EXPERIMENT}

A photograph of the experimental setup is shown in Figure 2. The source laser is a LSI pulsed 10.6 $\mu \mathrm{CO}_{2} \mathrm{TEA}$ laser with a pulse length of $100 \mathrm{~ns}$, a pulse energy of $150 \mathrm{~mJ}$, and a repetition rate less than $20 \mathrm{~Hz}$. The laser is incident on a $1 \mathrm{~m}^{2}$ surface of dry sand about $60 \mathrm{~cm}$ deep in which we would bury detectors or various types of subsurface targets. In the present studies of the opticalacoustic conversion mechanism, the acoustic signal was measured with a wide-band hydrophone $(0-100 \mathrm{kHz})$ which was buried at various depths and positions in the sand. This configuration contrasts with the experimental setup that we used to image underground objects. For imaging, our best results were obtained with a narrow-band $(30 \mathrm{kH})$ tuned PZT detector which was suspended in the air above the position of the laser pulse on the surface of the sand.

The laser was used in two configuration: unfocused or with a focusing lense to reduce the spot size. The unfocused laser spot is an oval approximately $1.1 \times 0.7 \mathrm{~cm}$ in size. A germanium lens with a focal length of $25 \mathrm{~cm}$ was used to focus the beam to a spot size less than $1 \mathrm{~mm}$ in diameter, comparable to the size of the sand grains. $10.6 \mu$ light is very effectively absorbed in soil with a reflection less than $10 \%$ and skin depth of approximately $5 \mu$. The sound produced by both the focused and the unfocused beams was audible across the room. With the focused beam the sound was considerably louder and there were flashes of visible light emitted and some movement of sand grains at the spot where the laser hit the surface.

The acoustic signal detected by the buried hydrophone for the focused and unfocused beams is shown in Figure 3 and Figure 4.

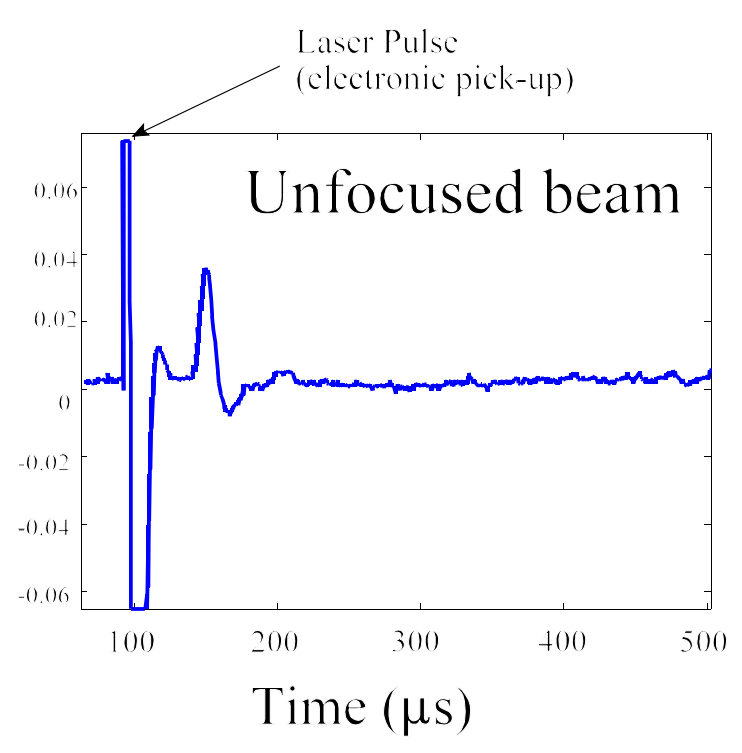

Figure 3 The time trace of the acoustic signal measured by a buried hydophone as a result of a pulse from the unfocused laser on the sand surface.

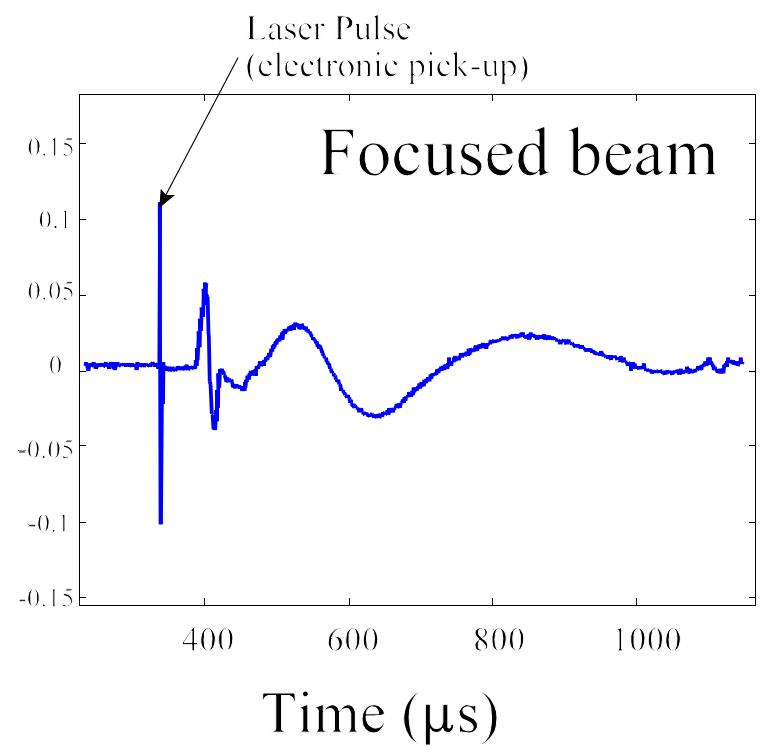

Figure 4 The time trace of the acoustic signal measured by a buried hydophone as a result of a pulse from the focused laser on the sand surface. 


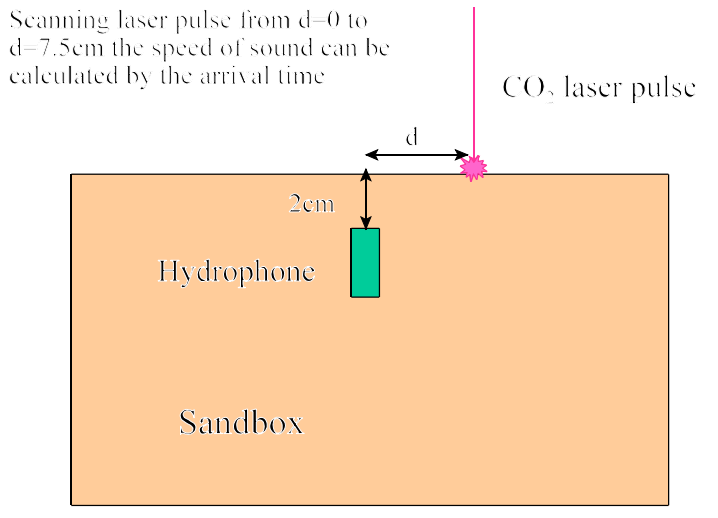

Figure 5 Experiment to measure the velocity in sand of the acoustic features in Figure 3 and 4.

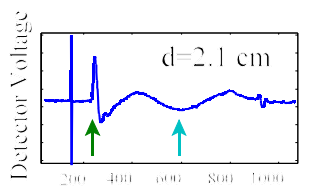

Time $(\mu s)$
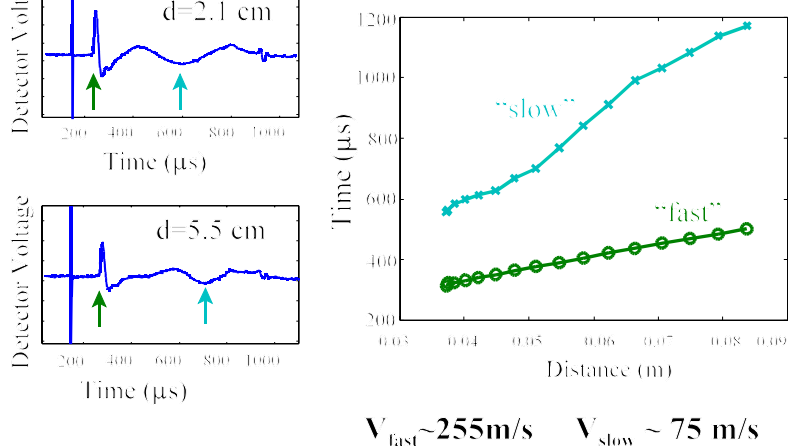

Figure 6 The arrival time $v s$. distance for the features indicated by the arrows in the inset. The propagation velocity in sand of the two features is determined as indicated from the slope of the lines.

The initial spike is electrical noise caused by the laser discharge. The acoustic pulse is dominated by a sharp peak with a width (about $10 \mu \mathrm{s}$ ) that is probably limited by the bandwidth of the $100 \mathrm{kHz}$ detector. For the focused beam only, there is a second, much broader feature with a peak-to-peak width of about $300 \mu \mathrm{s}$.

To determine the propagation speed of these two different acoustic features observed with a focused laser beam, we measured the change in the acoustic signal as a function of distance from the laser spot to the detector using the configuration in Figure 5 The time trace of the acoustic signal measured by a buried hydophone as a result of a pulse from the focused laser at two different positions on the sand surface are shown on the left in Figure 6. Identifying the sharp feature by the initial spike, and the broad feature by the dip between the two broader peaks, in Figure 6 we plot the time delay after the laser fires of these two features as a function of distance from the laser spot to the detector. By taking the slope of these two curves, we find the velocities of the two features are dramatically different. The sharp feature propagates with a velocity around $255 \mathrm{~m} / \mathrm{s}$, while the broad feature propagates with a velocity of about $75 \mathrm{~m} / \mathrm{s}$.

The observation of acoustic modes with two different velocities in porous media is a well-known phenomena. In the theory of Biot, the solution for acoustic propagation in a random porous elastic media gives rise to two modes: a mode in which the frame and the fluid move in phase (compressional wave) and an addition mode in which the motion of the frame is out of phase with the motion of the fluid. Typically the in-phase mode has a velocity which is close to the velocity of sound in the fluid, while the out-ofphase mode, the "Biot slow wave" has a lower velocity. If we tentatively make an identification of the features we observe with the Biot modes, the sharp feature which travels at $255 \mathrm{~m} / \mathrm{s}$, close to the velocity of sound in air, could be the Biot in-phase mode, while it is natural to identify the broader feature, low-velocity feature with the Biot slow wave. Note that we only observe this second, slower wave under excitation with a focused laser beam.

To measure the angular dependence of the magnitude of the acoustic signal from the focused beam, we positioned hydrophones at different angles along an arch buried in the sand centered on the position of the laser spot, as shown in Figure 7. In Figure 8 and 9, we plot the peak amplitude of the sharp "fast-wave" signal and the

\section{Angular Distribution of Acoustic Intensity}

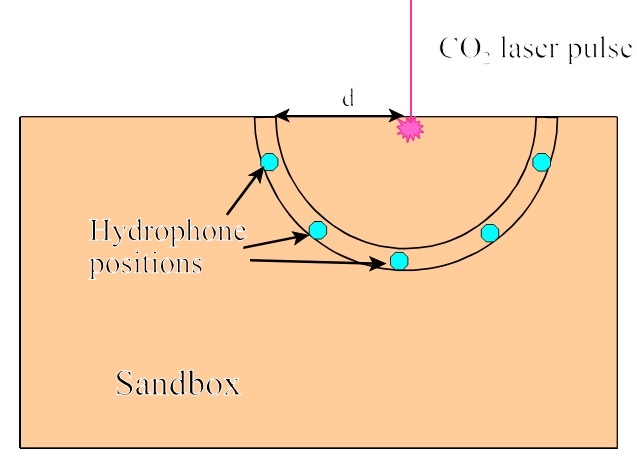

Figure 7 Experimental setup to measure the angular directivity of the acoustic features in Figure 3 and 4. 
Fast Wave Angular Distribution (Focused laser beam)

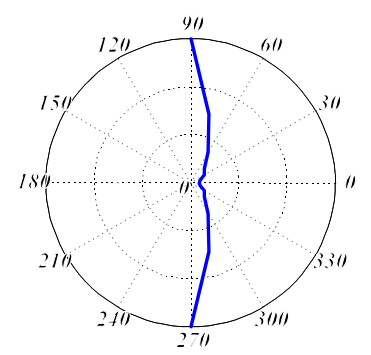

Rayleigh distance $=D^{2} / \lambda \leq 0.01 \mathrm{~cm}$
Slow Wave Angular Distribution (Focused laser beam)

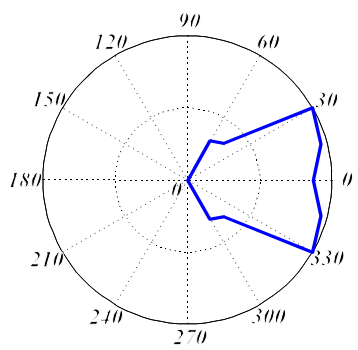

Figure 9 Angular dependence the amplitude of the "slow wave" feature produced with a focused laser beam.
Figure 8 Angular dependence the amplitude of the "fast wave" feature produced with a focused laser beam.

broad "slow-wave" signal as a function of angle from the normal. The two modes show a dramatic difference in angular distribution, with the slow wave signal propagation peaked at angles perpendicular to the surface, while the fast-wave signal strength is peaked along the surface of the sand. The angular dependence of the fast-wave mode is hard to understand, since when the beam is unfocused we observe only a single mode with a shape and velocity similar to the fast-wave signal, but with an angular distribution sharply peaked at a direction normal to the surface, as shown in Figure 10.

The unfocused beam has a Rayleigh length $\mathrm{d}^{2} / \lambda$ on the order of $1 \mathrm{~cm}$, so for distances into the sand on the order of $1 \mathrm{~cm}$ the unfocused beam looks like an extended source. The focused beam, on the other hand, has a Rayleigh length less than a millimeter and is a point source to a good approximation. Since the radius of the detector arch was about $10 \mathrm{~cm}$, it is unlikely, however, the difference between the fast-mode angular dependence seen in Figure 9 and 10 can be accounted for by such physical optics effects. It is more likely that the different acoustic excitation physics between the broad laser beam and the focused beam, such as illustrated in Figure 1, may account for these differences. We will return to this question later.

Table I summarizes the differences between the two acoustic modes that are observed with the focused laser beam. We note that these differences may impact on the utility of the two modes for underground detection and imaging. The fast mode, having a

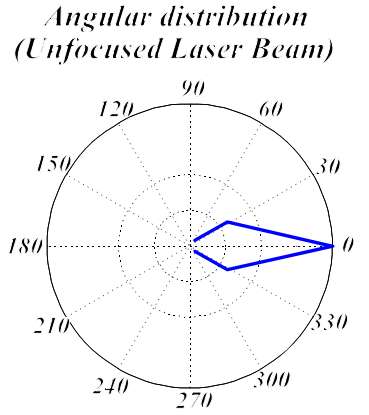

Rayleigh distance $=D^{2} / \lambda \_1 \mathrm{~cm}$

Figure 10 Angular dependence of the amplitude of the fastwave feature produced by an unfocused laser beam.
Table I: Comparison of Fast-Wave and Slow-Wave laser-induced acoustic features produced with a focused laser beam.

Comparison of Fast and Slow Acoustic Waves

\begin{tabular}{|l|l|l|}
\hline & Fast Wave & Slow Wave \\
\hline Unfocused Beam & Yes & No \\
\hline Focused Beam & Yes & Yes \\
\hline Speed & $250 \mathrm{~m} / \mathrm{s}$ & $75 \mathrm{~m} / \mathrm{s}$ \\
\hline Frequency peak & $100 \mathrm{kHz} *$ & $3 \mathrm{kHz}$ \\
\hline Directivity & $\begin{array}{l}\text { Along } \\
\text { Surface }\end{array}$ & $\begin{array}{l}\text { Perpendicular } \\
\text { to Surface }\end{array}$ \\
\hline$\alpha(\omega)$ & Unknown & Unknown \\
\hline
\end{tabular}

$*$ Band-width of detector $100 \mathrm{kHz}\left(1 / \mathrm{At}_{\text {twaer }}=10 \mathrm{MHz}\right)$ 
higher spectral frequency content may be more useful for highresolution imaging where a small wavelength is desired. On the other hand, if we have correctly identified the slow wave as a Biot out-of-phase wave, it will see an exceptionally large contrast between the porous soil where the slow wave is allowed and any subsurface non-porous object where, since there is no longer any differentiation between frame and fluid, its propagation is forbidden. There may also be implications about detection strategies, since to the extent that the energy of the slow-wave is carried in the fluid, techniques that measure the motion of the solid surface, such as laser vibrametry, will be less effective than with an ordinary compressional wave. On the other hand, for the same reason the mismatch between the poro-elastic wave and the wave in the air may be less for the slow-wave than the fast wave, so that detection by means of a microphone above the soil may be more favorable for the slow wave.

It is noteworthy that our attempts so far at subsurface imaging with the focused beam have been less successful than our previous work with the unfocused beam. These imaging studies were carried out with an acoustic detector suspended in the air above the surface. It is not possible to say at present whether the lack of success was due to an inherent effect such as the longer wavelength of the slow wave, less efficient acoustic coupling to or from the soil and air, or some interference effects between the fast and slow acoustic waves, or more incidental effects such as noise from the movement of sand grains and drifting of the laser focus due to heating of the air and movement of the surface.

One clue about the mechanism for acoustic excitation is that a flash of visible light is observed which the $\mathrm{CO}_{2}$ pulse hit the surface of the sand when the beam is focused, but not for the unfocused beam. Origins of this visible light that we considered were blackbody incandescence of the sand surface, photoexcitation and luminescence of the sand material, and electric field ionization and recombination. The energy density of the focused beam is sufficient to heat the sand over $10^{6}{ }^{\circ} \mathrm{C}$ if the effects of radiant emission and conduction are neglected, but this does not seem likely on the relatively long $(0.1 \mu \mathrm{s})$ timescale of the pulse. Luminescence of $\mathrm{SiO}_{2}$-based materials is usually very broad-band and can extend into the ultraviolet.

To gather more information about the light emitted when the focused $\mathrm{CO}_{2}$ laser pulse strikes the sand surface, we measured the spectrum of the emitted light using a multispectral filter and a photomultiplier detector as in Figure 11. The measured spectrum is reproduced in Figure 12 and Figure 13. The emission peaks are negative, because the electron current of the photomultiplier

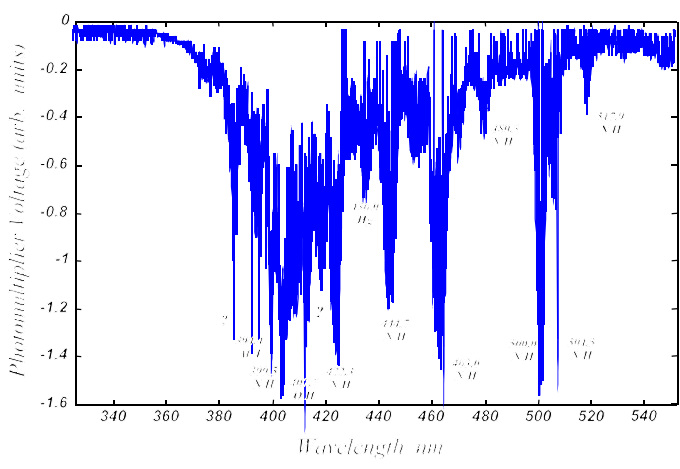

Figure 12 Spectrum of visible light produced by focused laser beam incident on surface of sand (short wavelength). Emission lines are identified from standard handbooks.

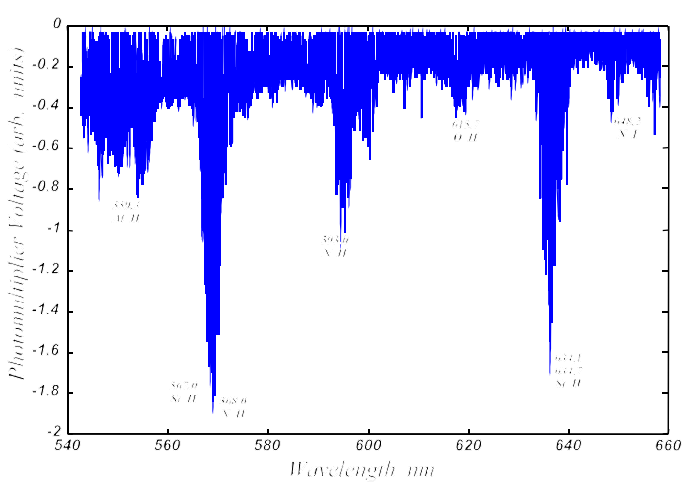

Figure 13 Spectrum of visible light produced by focused laser beam incident on surface of sand (long wavelength). Emission lines are identified from standard handbooks. 
is converted to a negative voltage by a voltage-to-current amplifier. Electron photocurrent resulting from the detected light yields a negative voltage.

We have identified emission lines in the spectrum by comparison with the CRC Handbook of Physics and Chemistry. We note that large emission lines associated with ionized nitrogen, oxygen, and silicon are present in the spectrum which implies that the model for optical field ionization is the best model for the production of the light. The concentration of the electric field of the optical signal near the sharp points of the sand grains is the most likely mechanism for the breakdown and ionization of the air and sand.

The acoustic production for the focused beam can then be best explained by the collapse of the air following the rapid expansion associated with the ionization and plasma formation as shown in Figure 14. The intense audible noise associated with the focused beam is analogous to the crack associated with an electrical arc or with thunder. One implication of this model is that the sound production for the focused beam is probably primarily as a compressional wave in the air above the soil. The reduced effectiveness of imaging with the focused beam may be a result of the poor coupling of this acoustic compressional wave in air into the soil.

The unusual angular dependence of the compressional fast wave for the focused beam seen in Figure 9 may represent the more effective transport of acoustic energy in the air along the surface of the sand than propagation in the sand. In effect, the sound produced by this ionization and collapse travels through the air to a point close to the high-angle, near-surface sensor and then propagates only a short distance into the soil to the detector. Since the velocity of the fast wave is very near the speed of sound in air, it is very difficult to distinguish this propagation channel from propagation through the soil. The slow wave may not have the same channel available to it because a Biot wave will not propagate in a single component fluid such as the air.

\section{CONCLUSIONS}

The production of acoustic energy in soil by a pulsed $\mathrm{CO}_{2}$ laser has been studied. When the laser beam is focused to a spot size comparable to the soil grains, a much louder acoustic sound is observed and two different acoustic modes are detected in the soil. One mode, which we identify with a normal compressional acoustic wave has a velocity ( $255 \mathrm{~m} / \mathrm{s})$ close to the speed of sound in air, and a bandwidth of at least $100 \mathrm{kHz}$. The second mode, which we have tentatively identified as a Biot slow-wave phenomena, has a velocity of only $75 \mathrm{~m} / \mathrm{s}$ and a spectral peak near $3 \mathrm{kHz}$. By analyzing the visible light emitted by the focused laser beam, we conclude that for the focused laser pulse the acoustic energy is produced by ionization of the air and soil components, with a rapid expansion and subsequent collapse of the air column. Preliminary investigations have indicated that imaging of subsurface objects with the focused beam laser is not as successful as with a broader unfocused beam. This may be a result of the poor coupling of sound produced in air into the soil. Studies to measure the attenuation and dispersion of the acoustic signals produced by the focused and unfocused laser beams are underway.

\section{ACKNOWLEDGEMENT}

The research described here was supported by the DARPA and ARO under the Multidisciplinary University Research Initiative.

\section{REFERENCES CITED}

1. S. W. McKnight, C. DiMarzio, W. Li, D. O. Hogenboom, G. Sauermann, "Laser-Induced Acoustic Detection of Buried Objects," SPIE Transactions on Detection of Mines and Mine-like Objects Vol. 3392, p. 841-847 (1998).

2. S. W. McKnight, W. Li, and C. DiMarzio, "Imaging of Buried Objects by Laser-Induced Acoustic Detection,", SPIE Transactions on Detection of Mines and Mine-like Objects, Vol. 3710, 231-238 (1999).

3. M. A. Biot, "Generalized Theory of Acoustic Propagation in Porous Dissipative Media,” J. Acoust. Soc. Am. 34, 179191 (1962). 